\title{
Two-drape closed pocket technique: minimizing aerosolization in mastoid exploration during COVID-19 pandemic
}

\author{
${\text { Arindam } \operatorname{Das}^{1}\left[(1) \cdot \text { Sandipta Mitra }^{1}(\mathbb{0}) \cdot \text { Soutrik Kumar }\right.}^{1} \cdot$ Arunabha Sengupta $^{1}$
}

Received: 26 July 2020 / Accepted: 2 September 2020 / Published online: 10 September 2020

c) Springer-Verlag GmbH Germany, part of Springer Nature 2020

\begin{abstract}
Background Mastoidectomy is associated with extensive bone-drilling which makes it a major aerosol generating procedure. Considering the ongoing COVID-19 global pandemic, it is essential to devise methods to minimize aerosolization and hence ensure safety of the healthcare workers during the operative procedure.

Methods Two disposable surgical drapes are used to create a closed pocket prior to commencement of mastoid bone-drilling. This limits aerosolization of bone-dust in the external operating theatre environment.

Conclusion Two-drape closed pocket technique is an easy, cost-effective and safe method to limit aerosolization of tissue particles during mastoidectomy.
\end{abstract}

Keywords Mastoidectomy · COVID-19 - Severe acute respiratory syndrome coronavirus 2

\section{Description of technique}

The patient position is supine with the head turned to the opposite side, supported. After routine antiseptic dressing of the surgical site, the site is made completely dry to ensure firm adherence. The surgical site is then draped with Surgiwear Major Drape. Full coverage of the head and neck area with firm adherence of the drape to the surgical site is ensured (Fig. 1). The incision area of the drape is then cut to expose the surgical site. The routine steps of mastoidectomy are then followed till the soft tissue work is complete. The second drape is installed before the start of bone-work. We have chosen a similar drape but customised it to fit in the optical lens of the operating microscope. A cut-out of an X-ray film of the exact diameter of the optical lens of the operating microscope (standard $250 \mathrm{~mm}$ for otological procedure) was created. This acted as a template for the exact size of the aperture. The second drape was cut accordingly

Electronic supplementary material The online version of this article (https://doi.org/10.1007/s00405-020-06353-5) contains supplementary material, which is available to authorized users.

Sandipta Mitra

onlysandipta@gmail.com

1 Institute of Post Graduate Medical Education and Research and SSKM Hospital, 244 AJC Bose Road, Kolkata 700020, India and fitted to the optical system of the microscope snugly (Figs. 2, 3). The rest of the drape surrounded the previouslydraped operating field with the edges infolded one atop the other together and sealed with a standard skin stapler. This created a sealed pocket in between the two drapes. The surgeon's working port lies between the two drapes. The surgeon can slip in his hands below the outer drape and over the first drape for manipulation. A small assistant's port is created laterally for instrumentation and additional irrigation, if required (Fig. 4). Another port is created laterally, for high-powered suction operating within the pocket (Fig. 4). All the aerosolized bone dust and spilled irrigation fluid are collected in this pocket (Fig. 5). This avoids contamination of the operating surgeon, assistant as well as the anaesthetist who are close by. Suction-irrigator was used during the bone-work. For demonstration purpose, the internal view of the pocket was recorded using a TEAC recorder (UR4MD). Certain precautions are adopted during removal of the assembly. The two drapes are removed together, without opening the pocket in order to contain the accumulated particles inside, keeping the suction connected at all times during removal to minimize external leak through the ports. After removal, the entire assembly with the suction tubing, is disposed as per the standard protocol for infected hospital waste disposal. 


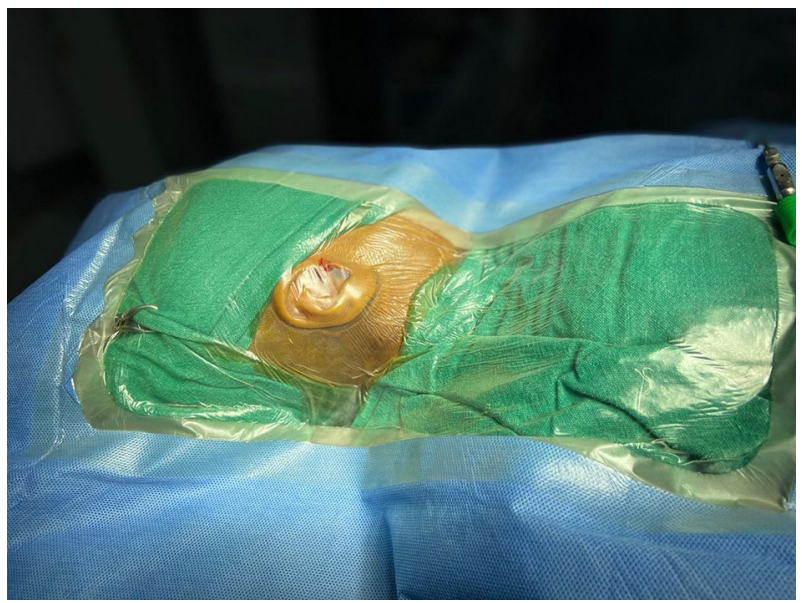

Fig. 1 The first drape firmly adherent to the surgical site

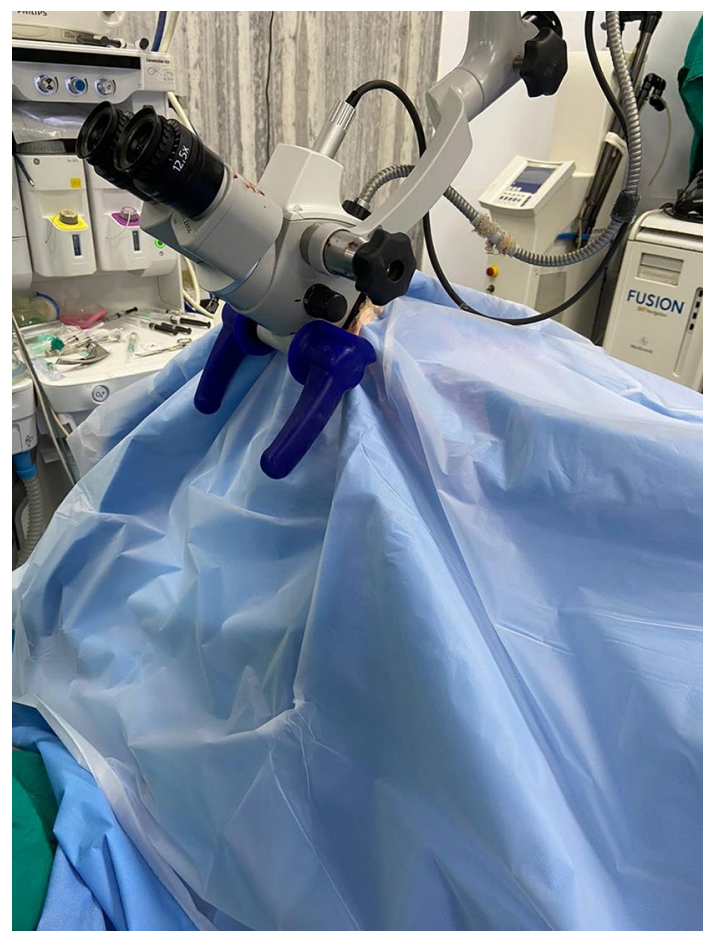

Fig. 2 The second drape fitted to the optical system of the operating microscope

\section{Indications}

The two-drape closed-pocket technique can be applied to any otological procedure, especially those involving major bone drilling, suction-irrigation with significant soft tissue aerosolization [1]. However, in the light of the ongoing pandemic, the indications of otologic surgery as per the British Society of Otology are restricted to mastoid

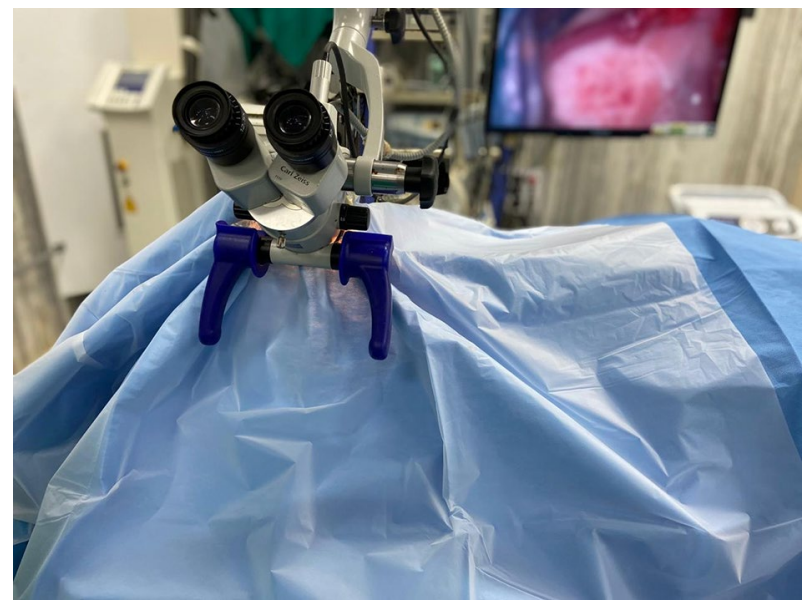

Fig. 3 External view of the closed pocket system

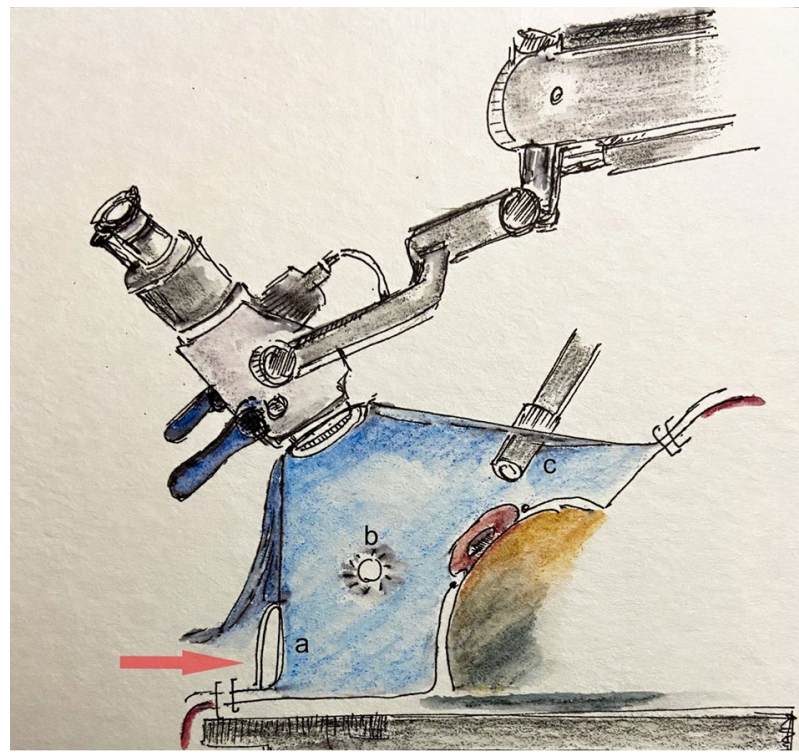

Fig. 4 Authors' illustration of the closed pocket system with a surgeon's working port (passage indicated by red arrow); b assistant's port; $\mathbf{c}$ port for high-powered suction for the pocket

exploration for the management of acute mastoiditis not resolving with conservative management, otogenic intracranial sepsis and operable temporal bone malignancy [2]. We have used this technique in 24 cases, of which 11 were cholesteatoma with intracranial complications, 6 were cholesteatoma presenting with facial nerve paralysis, 5 cases of recalcitrant mastoiditis not responding to medical therapy and 2 cases of temporal bone malignancy. 


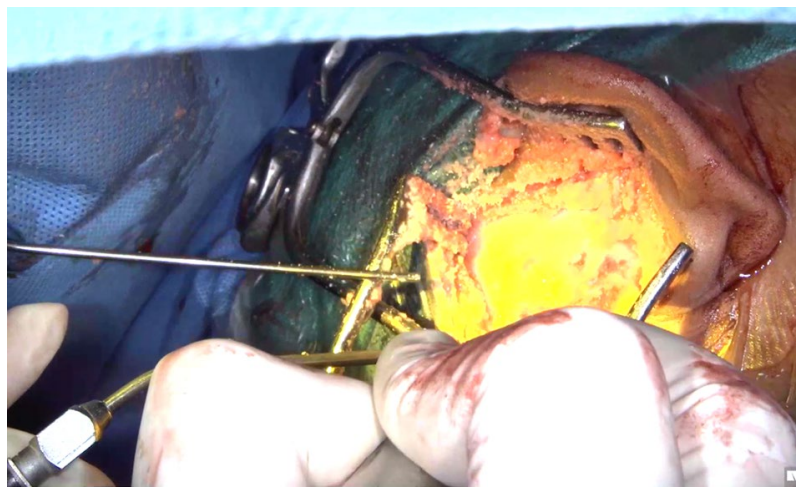

Fig. 5 Internal view of the closed pocket; Note the bone dust and irrigation fluid collected within the closed pocket

\section{Limitations}

The technique creates a closed pocket in which the aerosolized particles collect. However, this implies that the usual macroscopic visibility of the operating field, at large, is clouded and is associated with restriction of free manoeuvring for the operating surgeon and more so for the assistant. A clear second drape is a better option in this respect, to overcome this limitation. However, this advantage, by large, is only temporary and is soon lost after commencement of drilling, after the clear drape is soiled with spilled bone dust and irrigation fluid. Secondly, the technique is not an absolute protective barrier against exposure to aerosolized particles. This can only reduce the load of probable viral-infected tissues that a surgeon, assistant and the anaesthetist are exposed to during otologic surgery involving high-speed drilling and suctioning.

\section{How to avoid complications}

The loss of macroscopic visibility of the operating field during ear surgery may carry some risks. To name a few and avoid them one should always stop the burr under direct vision under the microscope and then slip it out gently from the pocket, if a change in the burr size is contemplated. Secondly, sharp micro-instruments such as picks or sickle-knives must be used very gently under direct vision and rash movements should be avoided to prevent injury to patient, the operating surgeon or the assistant.

\section{Specific peri-operative considerations}

For the drapes to firmly adhere to the surgical field, the area must be clean, dry, free from hairs. The aperture in the second drape must be fashioned out using the custom template to ensure a snug fit around the microscope.

\section{Discussion}

A number of viruses, including coronavirus, have been found to exist in the middle ear as well as nasopharynx during episodes of acute otitis media [3, 4]. Recently, Frazier et al. have confirmed the presence of SARS-CoV 2 colonization in the middle ear and mastoid in deceased COVID-infected patients [5]. Though it is our protocol to test for SARS-CoV 2 by RT-PCR prior to posting a patient for surgery, there are two pitfalls. Firstly, the test carries false negative rates between 2 and 29\% which implies a sensitivity of 71-98\% [6]. Secondly, the test results may not depict the COVID-19 status of the patient on the day the patient is being operated as there is a chance of acquiring the infection during the hospital stay, till the day of surgery, owing to the widespread community transmission in our country, at present. High-speed drilling has been shown to generate significant amounts of aerosol putting the operating theatre workers at risk $[1,7]$. Hence, proper compartmentalization, for collection of bone-dust, irrigation fluids and other tissue particles is imperative to protect the staffs. The technique devised by us is a simple, cost-effective, flexible, freely-movable, easilyachievable set-up that allows the surgeon or assistant to operate without being directly exposed to aerosolized particles. This in conjunction with proper surgical methods, judicious operating time and use of a high-powered suction can further reduce the infective tissue load generated around the field, during surgery. Chari et al. have devised similar technique for reduction of aerosolization during mastoidectomy. However, their study has been demonstrated on cadaveric model, with a limited drilling time of $60 \mathrm{~s}$ with restricted number of burrs, without change in instruments or irrigation settings, that are imperative during live surgery. Notwithstanding these limitations, they have demonstrated that additional barrier with drapes can effectively reduce aerosol dispersion during mastoidectomy [8]. Chen et al. have demonstrated similar results on cadaveric model with limited drilling time [7]. Our set-up is sterile, single-use and is discarded in the same way as per the standard protocol for infected hospital waste. However, this is not an absolute solution and that standard safety measures, such as, use of personal protective equipment (PPE) with a well-fitted FFP3 mask and adequate eye-protection must be adopted in all cases.

\section{Conclusion}

The two-drape closed pocket technique can contain the excess tissue spillage and hence minimize contamination during mastoid exploration. This may reduce the chances of the health-care staffs in the operating theatre from being 
exposed to significant amounts of infected aerosol generated during high-speed drilling and suctioning.

\section{Summary}

- Mastoidectomy requires high-speed bone drilling and suction-irrigation

- Both of these procedures generate copious amounts of aerosolized tissues

- Amidst the global pandemic of COVID-19, mastoidectomies are being done worldwide as a part of life-saving procedures such as, cholesteatoma with complications, operable temporal bone malignancies, etc.

- In spite of stringent pre-operative testing, RT-PCR for SARS CoV-2 carries considerable chances of false negative report and due to the delay between the day of the test and surgery, it is advisable to exercise extreme caution before every potential aerosol-generating procedure

- We have devised a two-drape closed pocket technique to minimize the exposure of the health-care staffs in the operating theatre to aerosol generated during mastoidectomy

- It is a simple, cost-effective, flexible, freely-movable structure that allows the surgeon or assistant to operate without being directly exposed to aerosolized particles

- It is a sterile, single-use set-up that can be discarded safely as per the standard hospital waste management protocols

- Though not an absolute solution, it is a feasible arrangement that can trap the major bulk of the aerosolized tissue particles generated during drilling and hence avoids direct exposure of the surgeon, assistant, anaesthetist and other operating theatre staffs during surgery

\section{Compliance with ethical standards}

Conflict of interest The authors declare that they have no conflict of interest.
Ethical approval Our research involves human participants and conforms to the principles of Declaration of Helsinki.

Informed consent Written, informed consent was obtained from all participants.

\section{References}

1. Jewett et al (1992) Blood-containing aerosols generated by surgical technique: a possible infectious hazard. Am Ind Hyg Assoc J $53: 228-231$

2. https://www.entuk.org/guidance-undertaking-otological-proce dures-during-covid-19-pandemic

3. Wiertsema SP et al (2011) High detection rates of nucleic acids of a wide range of respiratory viruses in the nasopharynx and the middle ear of children with a history of acute otitis media. J Med Virol 83:2008-2017

4. Heikkinen et al (1999) Prevalence of various respiratory viruses in the middle ear during acute otitis media. $\mathrm{N}$ Eng $\mathrm{J}$ Med 340:260-264

5. Frazier KM, Hooper JE, Mostafa HH, Stewart CM (2020) SARS$\mathrm{CoV}-2$ virus isolated from the mastoid and middle ear: implications for COVID-19 precautions during ear surgery. JAMA Otolaryngol Head Neck Surg 2020:e201922

6. Watson J, Whiting PF, Brush JE (2020) Interpreting a COVID-19 test result. BMJ 369:m1808

7. Chen J, Workman A, Chari D, Jung D, Kozin E, Lee D et al (2020) Demonstration and mitigation of aerosol and particle dispersion during mastoidectomy relevant to the COVID-19 era. Otol Neurotol (Publish Ahead of Print)

8. Chari DA, Workman AD, Chen JX et al (2020) Aerosol dispersion during mastoidectomy and custom mitigation strategies for otologic surgery in the COVID-19 era. Otolaryngol Head Neck Surg 2020:194599820941835

Publisher's Note Springer Nature remains neutral with regard to jurisdictional claims in published maps and institutional affiliations. 